\title{
Life Cycle Inventory Analysis of Recycling: Mathematical and Graphical Frameworks
}

\author{
Jun Nakatani \\ Department of Urban Engineering, The University of Tokyo, 7-3-1 Hongo, Bunkyo-ku, \\ Tokyo 113-8656, Japan; E-Mail: nakatani@env.t.u-tokyo.ac.jp; Tel./Fax: +81-3-5841-1279
}

Received: 4 July 2014 / Accepted: 4 September 2014 / Published: 10 September 2014

\begin{abstract}
A mathematical framework of the life cycle inventory (LCI) analysis in life cycle assessment (LCA) of recycling is systematically reviewed with the aid of graphical interpretation. First, the zero burden approach, which has been applied to LCI analyses of waste management systems, is theoretically justified in terms of relative comparison of waste management options. As recycling is a multi-functional system including the dual functions of waste management and secondary material production, the allocation issue needs to be handled in LCIs of recycling, and two forms of system expansion, i.e., the avoided burden and product basket approaches, have dominated to avoid the allocation problem. Then, it is demonstrated that conclusions derived from both approaches should mathematically be identical as far as system boundaries are correctly defined. A criticism against system expansion is also reviewed from the viewpoint of ambiguity of what-if scenarios. As an approach to this issue, market-based consequential LCA is discussed in the context of LCI analyses of open-loop recycling.
\end{abstract}

Keywords: zero burden approach; system expansion; avoided burden approach; product basket approach; what-if scenario; consequential LCA

\section{Introduction}

Integrated waste management is one of the holistic approaches to environmental management and resource management. Hence, waste management options should be systematically examined, and in this context appropriate application of life cycle assessment (LCA) is required [1]. Since the beginning of the 1990s, LCA has been used to analyze recycling and to compare it with other waste management options [2]. Several literatures have so far summarized and discussed the methodology for applying 
LCA to waste management and recycling [1,3-7]. Despite those theoretical developments, the allocation problem with regard to open-loop recycling seems to be still controversial $[7,8]$.

In this paper, a mathematical framework of the LCI analysis of recycling is systematically reviewed, focusing on both the "zero burden" approach and "system expansion". With the aid of graphical interpretation, it is demonstrated that two forms of system expansion, i.e., the so-called "avoided burden" and "product basket" approaches, should derive mathematically identical conclusions as far as system boundaries are correctly defined. Then a criticism against system expansion is also reviewed from the viewpoint of ambiguity of "what-if" scenarios. As a comparably novel approach to this issue, market-based "consequential" LCA is reviewed and discussed.

\section{Method: Life Cycle Inventory Analysis}

The ISO standard (ISO 14040:2006) defines LCA as a tool to assess the potential environmental impacts and resources used throughout the product life cycle, i.e., from raw material acquisition, via production and use phases, to waste management including disposal as well as recycling [8]. The product life cycle is often mentioned as "from the cradle to the grave". According to the ISO standards, an LCA study consists of the following four phases:

- Goal and scope definition: the reasons for carrying out the study, the intended application and audience are described, and the system boundaries and the functional unit of the study are defined.

- Life cycle inventory (LCI) analysis.

- Life cycle impact assessment (LCIA): the magnitude and significance of the potential environmental impacts of the studied system are evaluated.

- Interpretation: the results from the previous phases are interpreted in terms of the goal and scope of the study to reach conclusions and recommendations.

Among those phases, LCI analysis makes a model of the product life cycle, relates inputs and outputs of processes to the functional unit, and then compiles all the relevant interventions, namely environmental burden emissions and resource consumptions. LCI compilation is divided into several methods: "process-based" analysis and "input-output (IO)-based" analysis [9], as well as three different forms of "hybrid" analysis [11]. The following discussions in this paper mainly target the process-based LCI, while the fundamental framework is valid for "tiered" hybrid analysis, where foreground systems including use and disposal phases are dependent on process-based LCIs and the use of IO-based LCIs is limited to background systems.

\subsection{Zero Burden Approach}

In LCIs of products, in principle, inputs and outputs crossing the system boundary should be flows that are extracted from the environment and flows that are emitted or discarded to the environment, respectively. Those inflows and outflows are referred to as "elementary flows" [10]. However, it is typically not true for LCA studies of waste management systems. Instead, the inputs are often regarded as waste products because inflows which are identical in all product systems to be compared can be disregarded [4]. This exercise is sometimes referred to as the "zero burden" approach, which takes the waste to be managed by the system as a given [5]. In this approach, the "cradle" is regarded as the 
point of waste generation where the product becomes waste, e.g., the dustbin in households. In addition, as a result of applying the zero burden approach, the functional units should be different between LCIs of products and waste: the former is defined in terms of the output of the system, i.e., the product, and the latter is defined in terms of the input of the system, i.e., the waste [5].

The zero burden approach can theoretically be justified with the aid of graphical interpretation as illustrated in Figure 1. The entire product life cycles are presented above, and all of them fulfill the function as production of one unit of the product. On the other hand, the below systems are composed of the minimum essential processes to compare the environmental burdens of different waste disposal options. All the below systems fulfill the same function, i.e., disposal of one unit of the waste product. Although the absolute values of LCIs would be different between the above and below systems, the comparative environmental burdens of respective options are consistent.

Figure 1. Theoretical system boundaries of LCIs of waste disposal options. The below systems disregard inflows which are identical in all the product systems. The functional units of respective systems are defined in terms of the shaded products or waste products.

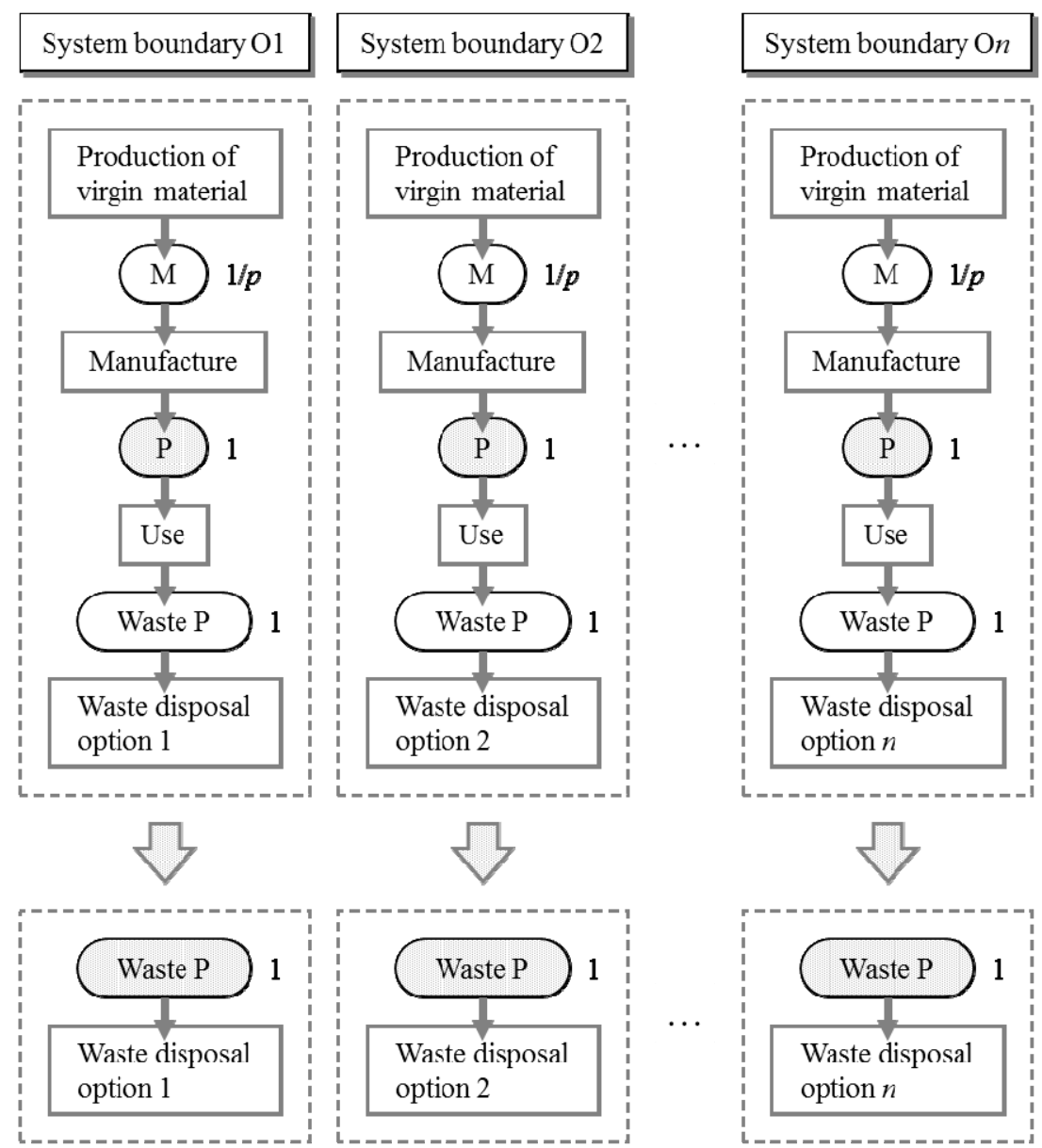

Note: $\mathrm{M}$ and $\mathrm{P}$ represent a material and a product, respectively. $p$ denotes the yield ratio of a manufacture process. Fine dashed lines denote the system boundaries.

\subsection{System Expansion: Two Approaches}

In LCI analyses including the so-called "multi-functional" processes, i.e., processes that yield more than one functional flows [7] and are shared between multiple product systems, the 
environmental burdens need to be allocated over the different functions. There are three types of allocation problems $[7,8]$ :

- Multi-output: having more than one functional outflows and no functional inflow, in which a single process produces multiple products (e.g., a refinery).

- Multi-input: having no functional outflow and more than one functional inflow, in which a process receives multiple waste products (e.g., a waste incinerator).

- Open-loop recycling: having one or more functional outflows and one or more functional inflow, in which a waste product is recycled into another product.

In other words, recycling is a multi-functional system including the dual functions of waste management and secondary material production. Allocation can be done on the basis of several principles such as physical and chemical causation, economic value, or other physical parameters including energy and mass [8]. However, those exercises are often not free from arbitrary choices, and the ISO standard recommends the following approaches to handle the allocation issue [8]:

- Subdivision [13]: dividing (partitioning) the process into sub-processes each with a single product, i.e., a number of "mono-functional" processes [7].

- System expansion [13]: expanding the system boundaries to include affected parts of other product life cycles in the system under study.

In LCIs of waste management systems in which recycling or energy recovery is included, system expansion has dominated over subdivision. System expansion in the broad sense can be classified into (a) the "avoided burden" and (b) the "product basket" approaches [14]. In the avoided burden approach, it is assumed that the recycled product or recovered energy from the investigated system will replace the competing product or energy source, and environmental burdens from the competing process are subtracted from those from the investigated system [8]. In the product basket approach, when a waste management option that includes recycling or energy recovery is assessed in comparison with a conventional option, the competing process producing the product or energy is added to the conventional system so that its entire function should be identical to the investigated system. In other words, the investigated system is "credited" with producing the equivalent amount of products or energy [4].

Theoretical system boundaries for the avoided burden approach are illustrated in Figure 2. Material recycling is generally categorized into either closed-loop recycling, where waste products are recycled into material that is used for products of the same kind, or open-loop recycling where they are recycled into material that is used for other kinds of products. The system boundaries A and B in Figure 2 represent the product life cycles where the waste products after use are processed by closed-loop and open-loop recycling, respectively. The system boundary $\mathrm{O}$ represents the case where the waste product is treated by a waste disposal option. The left-hand system boundaries include the entire life cycles of investigated products $\mathrm{P}$. In those systems, the upstream processes that all the product systems have in common can be canceled out on the basis of the zero burden approach described in the previous section. Besides, the downstream processes after the point where substitution occurs can be canceled out for open-loop recycling inside the system boundary. Then, those systems are simplified as illustrated in the right-hand system boundaries. They are composed of the minimum essential processes to compare the environmental burdens of closed-loop and open-loop recycling with waste 
disposal. Similarly, theoretical system boundaries for the product basket approach are illustrated in Figure 3. The upstream and downstream processes that all the systems have in common are canceled out in the right-hand (simplified) systems.

Those figures graphically present that, although the absolute values of LCIs are different between the entire and the simplified systems, the same conclusion should be drawn from both of them in terms of relative comparison among recycling and waste disposal options (see Figures 4 and 5). Moreover, by comparing the simplified systems between Figures 2 and 3, it is presented that the avoided burden and product basket approaches draw the same conclusions in terms of relative comparison. As shown in Figures 4 and 5, despite the different absolute values of LCIs between different approaches, the comparative environmental burdens of respective options are consistent.

As indicated by the simplified systems in Figures 2 and 3, it is often the case that a crucial factor for environmental consequences of recycling in system expansion is "what material competes with, and is eventually replaced by, recycled material from the investigated product" rather than the environmental burdens from the systems originally investigated. For instance, Bjorklund and Finnveden [2] conclude that for non-renewable materials the only crucial factor is what material is replaced. This leads to a criticism against system expansion in LCI analyses of recycling as reviewed in the next section.

Figure 2. Theoretical system boundaries of material recycling and waste disposal for the avoided burden approach. The system boundaries A, B and O represent closed-loop, open-loop recycling and waste disposal scenarios, respectively.

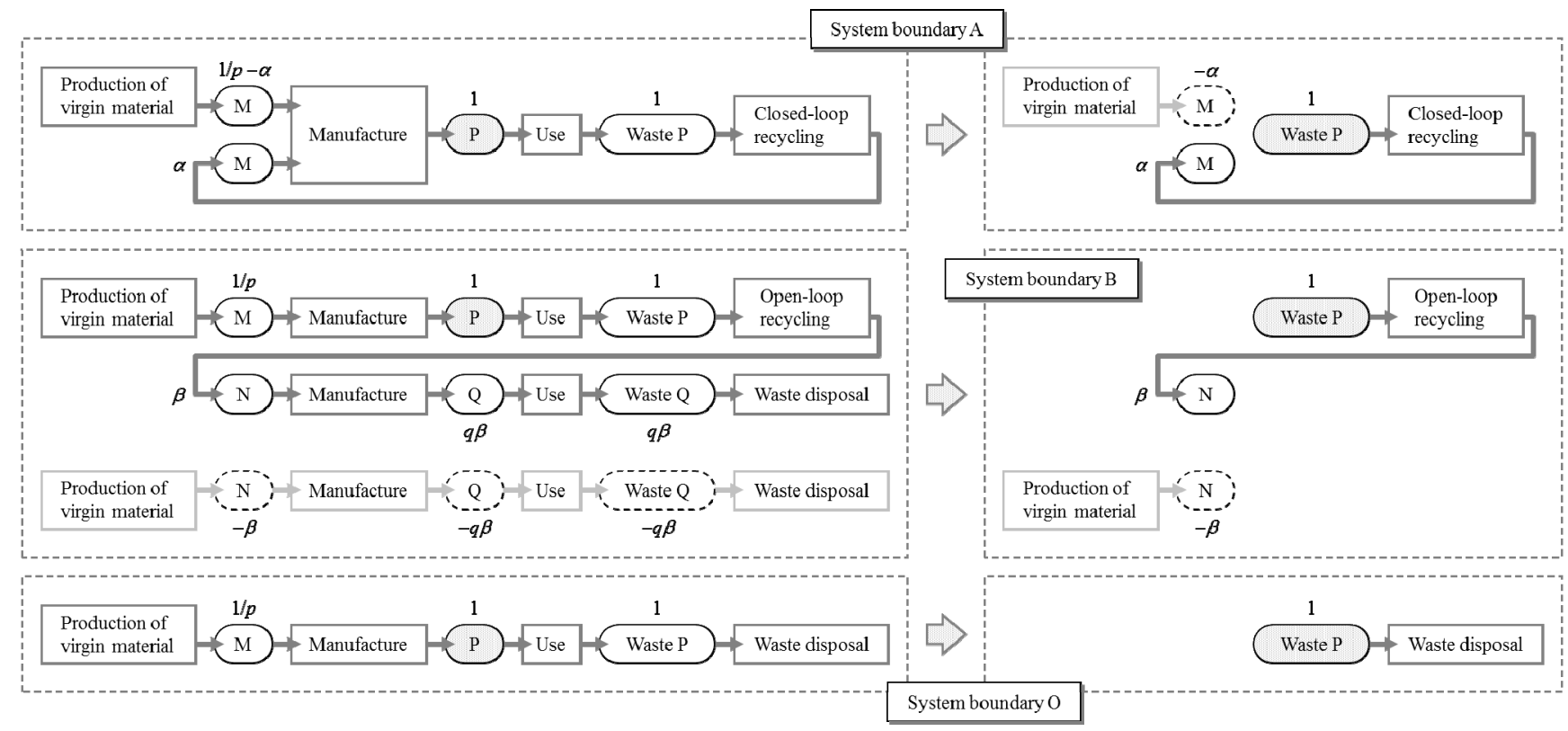

Note: $\mathrm{M}$ and $\mathrm{N}$ represent materials, and $\mathrm{P}$ and $\mathrm{Q}$ represent products. $\alpha$ and $\beta$ denote the yield ratio of closed-loop and open-loop recycling processes, and $p$ and $q$ denote the yield ratio of manufacture processes of products $\mathrm{P}$ and Q, respectively. Dark and light gray lines represent the life cycle of investigated product $\mathrm{P}$ and other kinds of products that are affected by a change in the recycling flows of the product $\mathrm{P}$, respectively. Materials and products in dashed circles are replaced by increased outflows of recycled material from the investigated product P. Fine dashed lines denote the system boundaries. Left-hand system boundaries can be simplified into right-hand system boundaries on the basis of the zero burden approach. 
Figure 3. Theoretical system boundaries of material recycling and waste disposal for the product basket approach. The system boundaries A, B and O represent closed-loop, open-loop recycling and waste disposal scenarios, respectively.

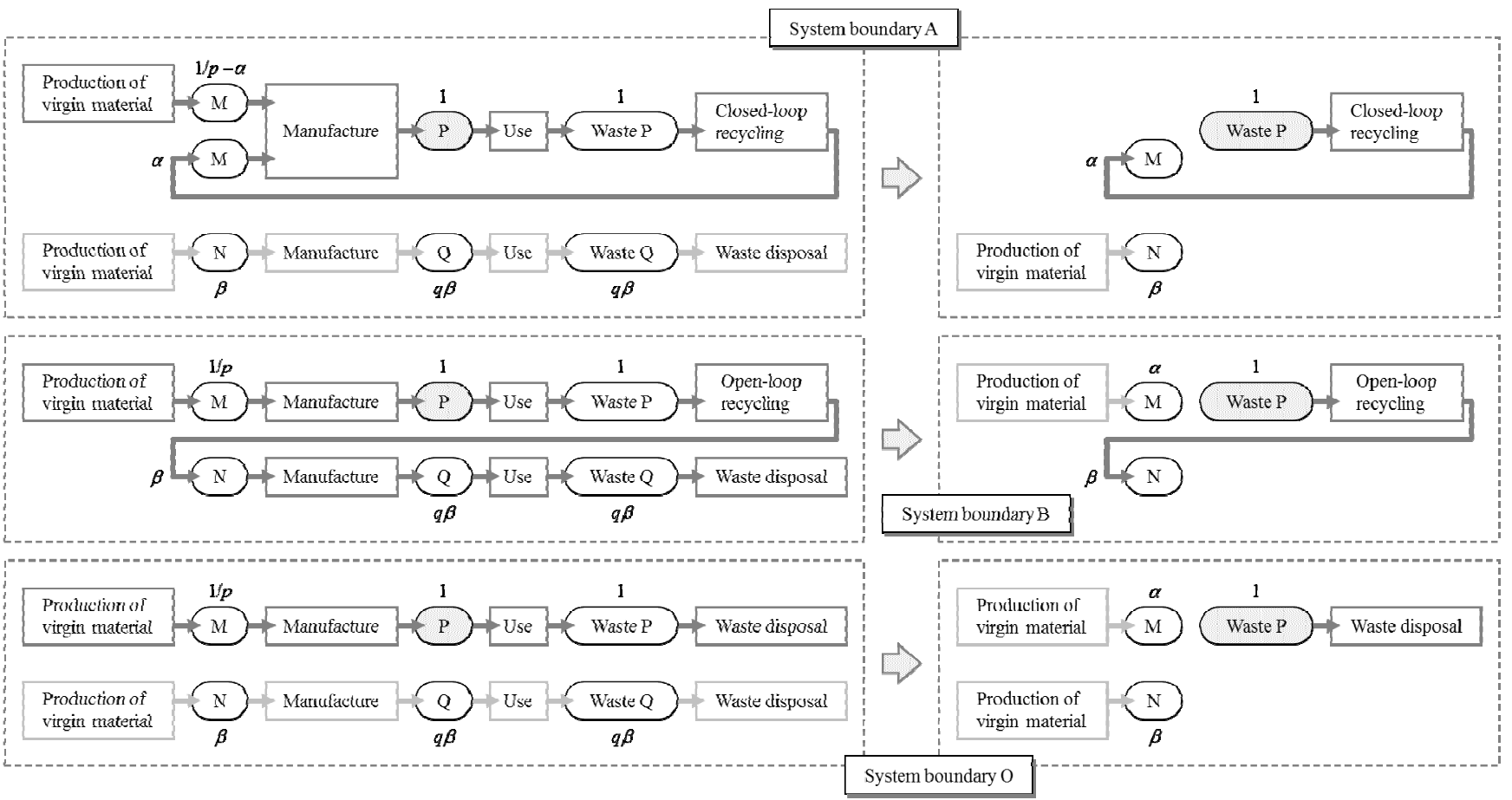

Note: $\mathrm{M}$ and $\mathrm{N}$ represent materials, and $\mathrm{P}$ and $\mathrm{Q}$ represent products. a and $\mathrm{b}$ denote the yield ratio of closed-loop and open-loop recycling processes, and $p$ and $q$ denote the yield ratio of manufacture processes of products $\mathrm{P}$ and Q, respectively. Dark and light gray lines represent the life cycle of investigated product $\mathrm{P}$ and other kinds of products that are affected by a change in the recycling flows of the product $\mathrm{P}$, respectively. Fine dashed lines denote the system boundaries. Left-hand system boundaries can be simplified into right-hand system boundaries on the basis of the zero burden approach.

Figure 4. An example of LCI analysis of recycling and waste disposal using the avoided burden approach (data adapted from Nakatani et al. [15]). A left-hand figure considers the entire life cycles including material production and manufacture of the investigated product (see the left-hand system boundaries in Figure 2), and a right-hand figure considers the simplified systems (see the right-hand system boundaries in Figure 2).
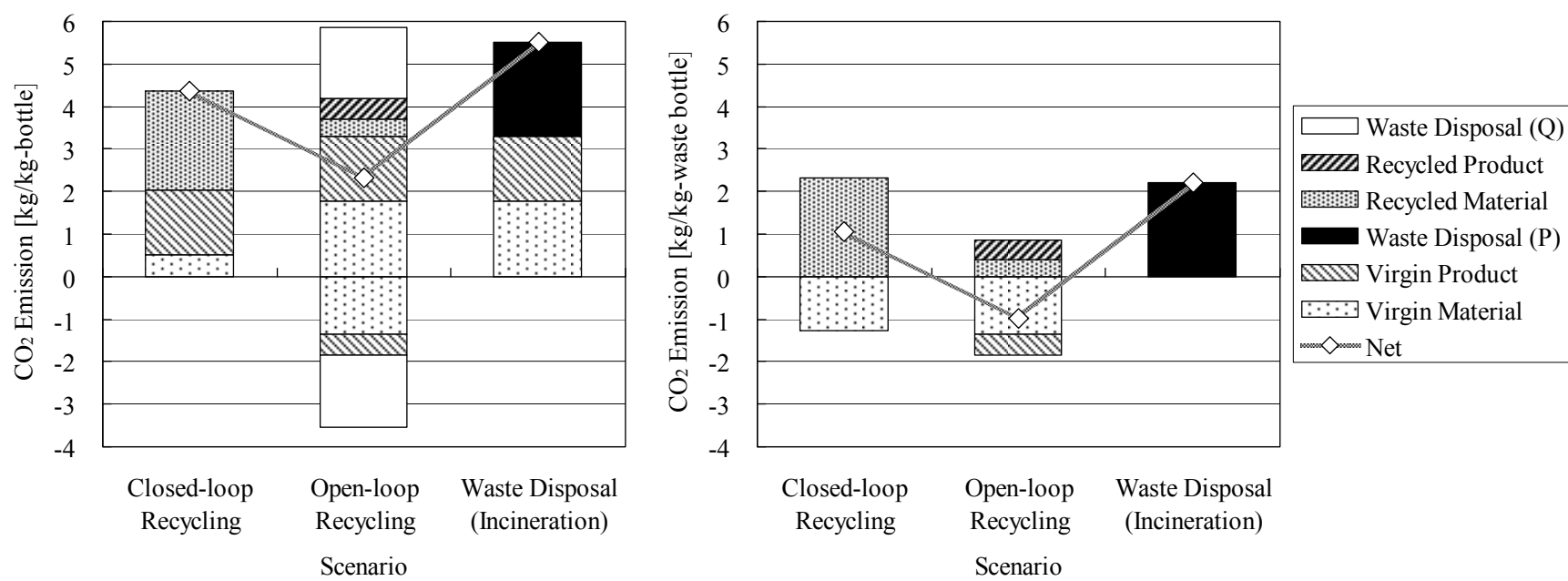
Figure 5. An example of LCI analysis of recycling and waste disposal using the product basket approach (data adapted from Nakatani et al. [15]). A left-hand figure considers the entire life cycles including material production and manufacture of the investigated product (see the left-hand system boundaries in Figure 3), and a right-hand figure considers the simplified systems on the basis of the zero burden approach (see the right-hand system boundaries in Figure 3).
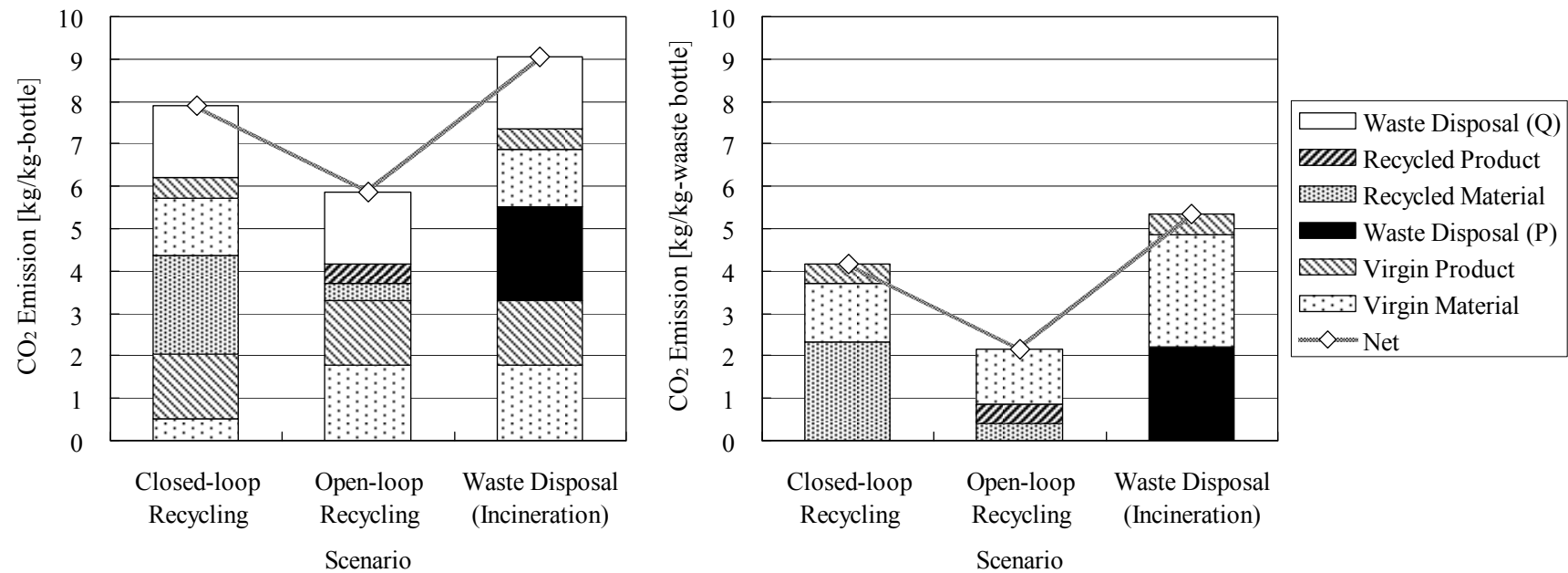

Mathematical frameworks for LCI of open-loop recycling hold true for other kinds of recycling such as feedstock recycling as well as energy recovery of waste. For those cases, the material $\mathrm{N}$ in Figure 2 or Figure 3 is regarded as recycled feedstock or recovered energy that would replace the competing feedstock or energy source (see Figure 6).

Figure 6. An example of a system boundary of energy recovery for the avoided burden approach. Electricity generated by energy recovery from waste incineration replaces public (grid) electricity supply including thermal, hydroelectric and nuclear power generation.

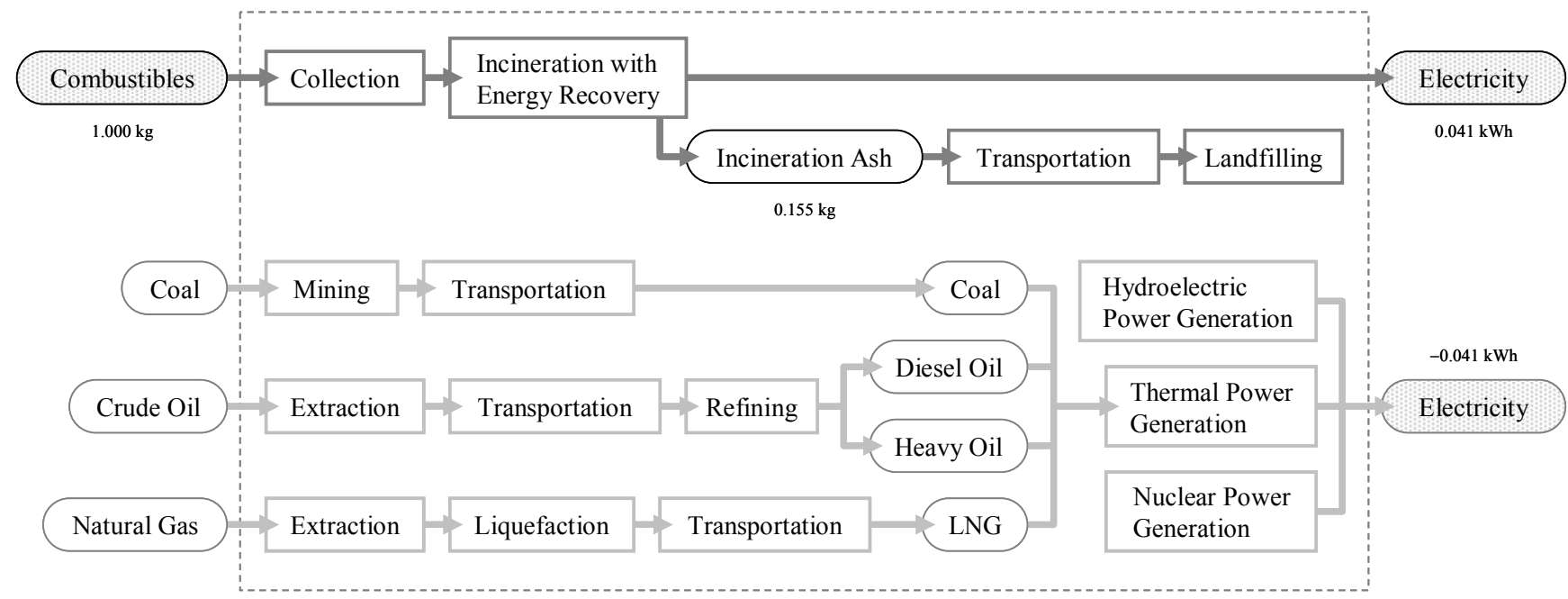

A typical elementary mistake of system expansion for recycling is, especially in the cases where the zero burden approach is concurrently applied, that environmental consequences of recycling are dependent on the number of recycling times, or recycling loops. This often misleads to conclusions 
that closed-loop recycling has an advantage over and open-loop recycling with regard to the environmental burdens "because of the infinite recycling loops". As easily understood with the simplified systems in Figures 2 and 3, closed-loop and open-loop recycling are mathematically indifferent in LCIs of recycling. In addition, as far as the destination of the recycled product is identical to that of the competing product, the environmental burdens after the point where substitution occurs can be canceled out inside the system boundary even when the recycled product is further recycled after use. This means the environmental consequences of open-loop recycling are also independent from the number of recycling times.

\section{Result: Ambiguity of What-If Scenarios}

As described in the previous section, system expansion seems a feasible approach to avoid allocation problems in LCIs of recycling. There are, however, some critical questions on system expansion, especially, specifications of material that is replaced by the recycled material [4]. In most LCA studies for open-loop recycling, it is implicitly assumed that a recycled product replaces a product of the same type, in particular (i) a product from virgin material. Besides, recycled material could also (ii) compete with recycled material from other kinds of products, or (iii) replace no material at all if it is used for functions that would otherwise not be generated [16]. It is also probable that recycled material replaces completely different types of material [16]. As illustrated in Figure 7, the mix of materials actually replaced by an increased outflow of recycled material depends on how the market reacts to it [16]. However, it is difficult to identify what processes are actually affected by a change in the recycling flows, and this issue would possibly fall into ambiguity of "what-if" scenarios.

Figure 7. Illustration of materials replaced by an increased outflow of recycled material.

The ratio among the what-if scenarios (i)-(iii) is dependent on the market.

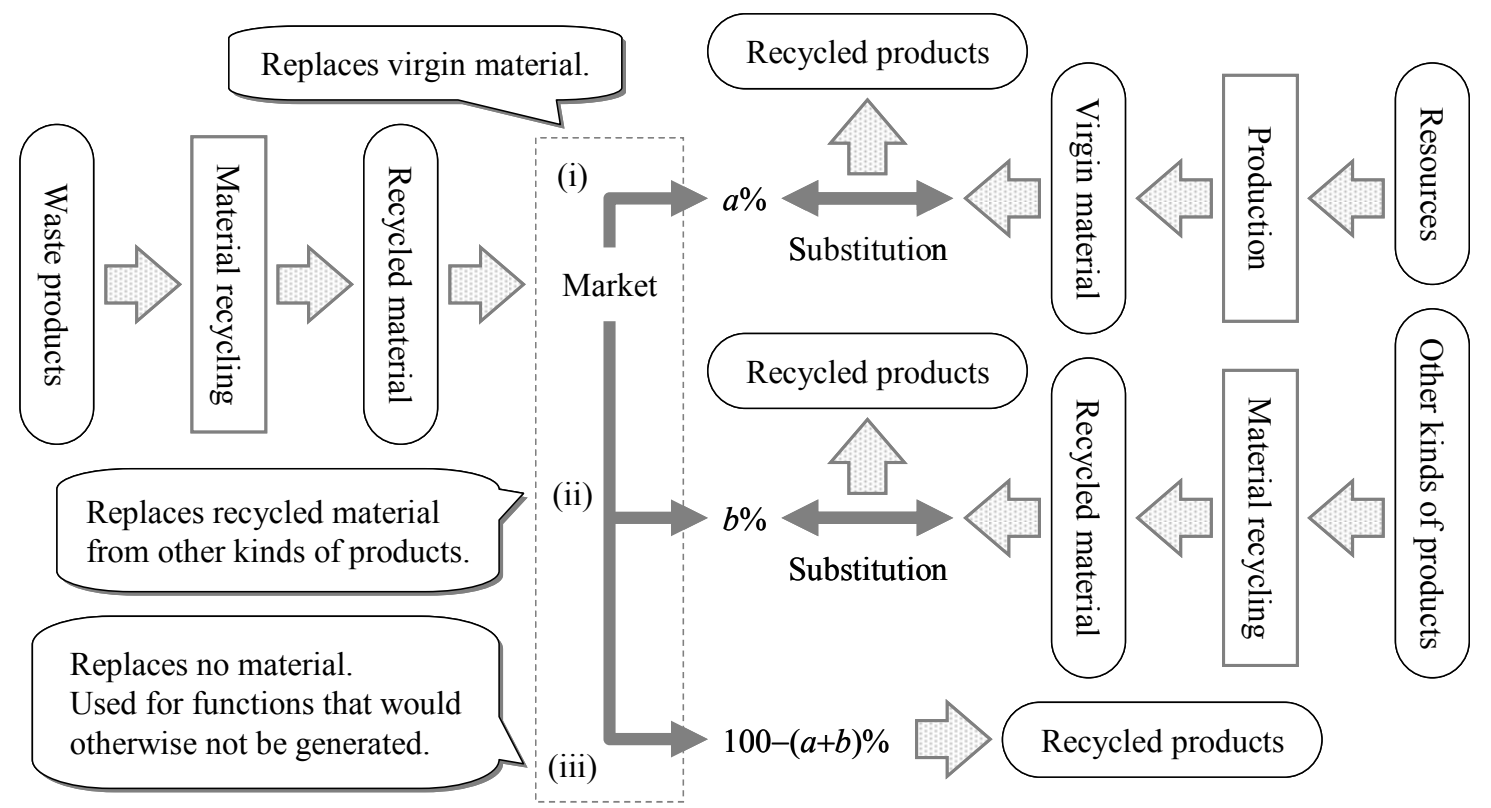

With regard to debates between the partitioning approach (subdivision) and the avoided burden approach (system expansion) to handle the multi-functional processes including recycling, Heijungs and Guinée [7] conclude that what-if scenarios should as much as possible be kept outside the LCA 
methodology itself, and LCA as a scientific tool should "stay to the facts" where there are facts. In system expansion, technological specifications of avoided processes should be made, which may make all attempts futile and lead to accumulation of what-if argumentations. The partitioning approach, on the other hand, always leads to a solution whenever the so-called "allocation factors" have been set, and has no specific theoretical problem [7], Thus, this approach is regarded as a conservative exercise where diverging results would be avoided. However, as also admitted by Heijungs and Guinée [7], the major practical problem is the specifications of the allocation factors, conventionally chosen on the basis of physical, economic or other parameters. Any choice of allocation principle is essentially arbitrary. In this sense, the fundamental problem of the partitioning approach is indifferent from the conventional allocation exercises. Although this approach is theoretically reasonable and free from ambiguity of what-if scenarios, further practical development would not be expected.

\section{Discussion on Consequential Life Cycle Assessment}

Recently, there is an increasing interest in the distinction between two types of LCA methods: attributional and consequential LCA [17]. Attributional LCA only accounts for physical flows involved in the product life cycle, whereas consequential LCA, on the other hand, aims to describe how physical flows can change as a consequence of an increase or decrease in demand for the product system under study [18]. Consequential methodology implies that allocation is avoided by means of system expansion, which describes how multi-functional processes including open-loop recycling affect processes outside the life cycle originally investigated [19].

As a comparably novel approach to the what-if scenario issue, market-based consequential LCA has been discussed in the context of LCIs of open-loop recycling. Ekvall [20] suggests a conceptual model that shows how a change in the recycling flows affects the market for other recycled material and for virgin material by utilizing the price elasticity of supply and demand (Figure 8). Ekvall and Weidema [19] refer to the model in the context of the consequential LCA of open-loop recycling.

Several case studies have been found to apply this conceptual model so far. In practice, simplifications are required to make the methodology operational. For example, Ekvall and Weidema [19] propose a simplification of the model to assume that recycled material competes only with (i) virgin and/or (ii) recycled material of the same type, where the effect of a change in the flows $\mathrm{X}$ and $\mathrm{Y}$ on the flows (i) $\mathrm{Z}$ and/or (ii) $\mathrm{W}$ is considered in Figure 8. On the basis of this simplification, Frees [21] applies the model to aluminum recycling and concludes that production of primary aluminum, rather than recycled aluminum, would be avoided because of the low price elasticity of supply of aluminum scrap. Nakatani et al. [15] also apply the model to plastic bottle recycling, by assuming that the recycled material replaces (i) virgin material of the same type and/or (iii) no material, where the effect of a change in the flows $X$ and $Z$ on the flows (i) $T$ and/or (iii) $E$ is considered in Figure 8. They conclude that, as far as a change in the outflows of recycled material from the investigated product does not affect the demand for the product originally investigated, closed-loop recycling, which certainly replaces virgin material of the same kind, has advantage over open-loop recycling, which possibly replaces no material. 
Figure 8. A conceptual model of closed-loop and open-loop recycling through the market (adapted from Ekvall [20]).

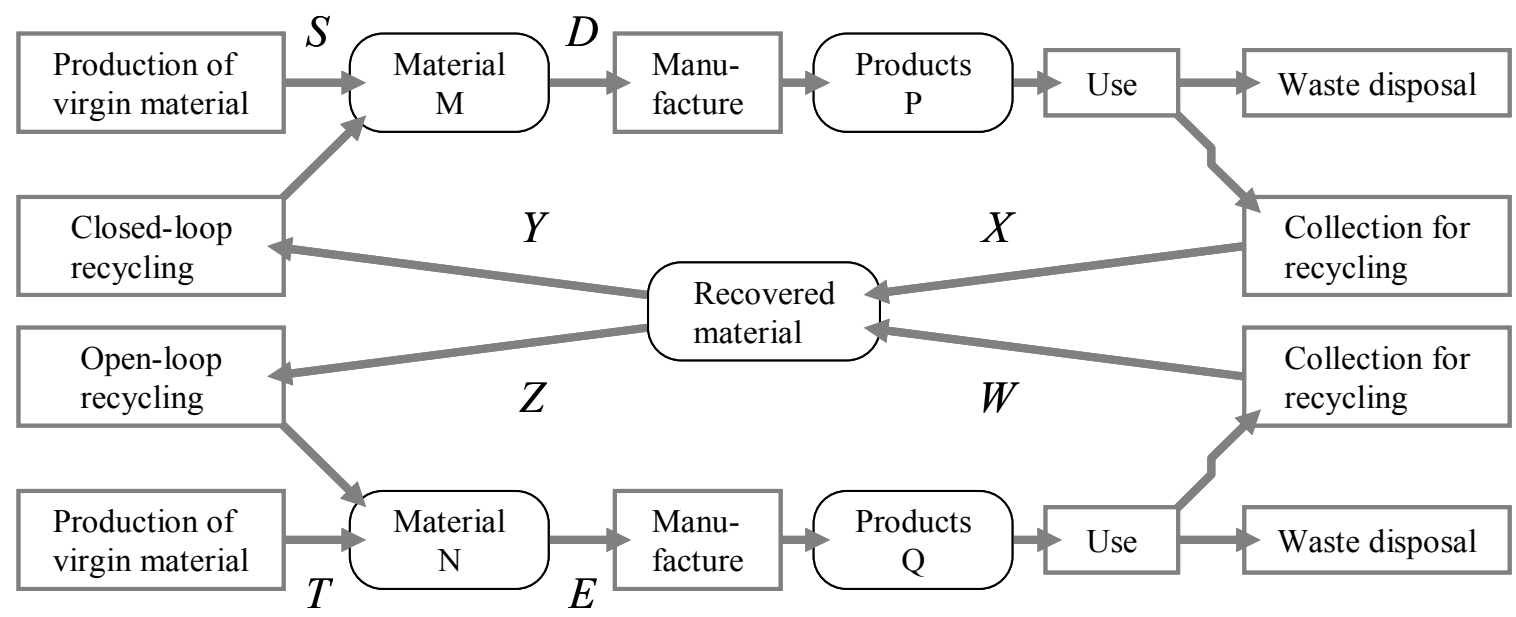

\section{Conclusions}

In this paper, with the aid of graphical interpretation, a mathematical framework of the LCI analysis of recycling was systematically discussed, focusing on system expansion. Although the model of market-based consequential LCA can be recognized as a challenging solution to handle the what-if scenario issue, the feasibility of the model is still unknown because of the lack of accumulation of case studies. In particular, it is doubtful if the price elasticity of demand and supply could be correctly estimated, while some case studies that apply the model have indicated environmental consequences of recycling are largely dependent on the estimation of price elasticity.

As discussed above, subdivision as a typical form of attributional methodology aims to avoid diverging LCI results by keeping uncertainty from spreading outside the product system originally investigated. On the other hand, system expansion as a basis of consequential methodology aims at assessing the environmental consequences that occur in social systems with a broader viewpoint. Further debates on the advantages and disadvantages of both approaches are required in the context of LCIs of recycling. In particular, comparative case studies applying both approaches concurrently should be helpful for methodology development.

\section{Acknowledgments}

The author gratefully appreciates fruitful discussions and friendship during the stay at the Institute of Environmental Sciences (CML), Leiden University.

\section{Conflicts of Interest}

The author declares no conflict of interest.

\section{References and Notes}

1. Clift, R.; Doig, A.; Finnveden, G. The Application of Life Cycle Assessment to Integrated Solid Waste Management: Part 1-Methodology. Trans. Inst. Chem. Eng. 2000, 78, 279-287. 
2. Bjorklund, A.; Finnveden, G. Recycling Revisited-Life Cycle Comparisons of Global Warming Impact and Total Energy Use of Waste Management Strategies. Resour. Conserv. Recycl. 2005, 44, 309-317.

3. Tillman, A.M.; Ekvall, T.; Baumann, H.; Rydberg, T. Choice of System Boundaries in Life Cycle Assessment. J. Clean. Prod. 1994, 2, 21-29.

4. Finnveden, G. Methodological Aspects of Life Cycle Assessment of Integrated Solid Waste Management Systems. Resour. Conserv. Recycl. 1999, 26, 173-187.

5. McDougall, F.R.; White, P.R.; Franke, M.; Hindle, P. Integrated Solid Waste Management: A Life Cycle Inventory, 2nd ed.; Blackwell Publishing: West Sussex, UK, 2001; pp. 103-128.

6. Ekvall, T.; Assefa, G.; Bjorklund, A.; Eriksson, O.; Finnveden, G. What Life-Cycle Assessment Does and Does Not Do in Assessments of Waste Management. Waste Manag. 2007, 27, 989-996.

7. Heijungs, R.; Guinée, J.B. Allocation and "What-if” Scenarios in Life Cycle Assessment of Waste Management Systems. Waste Manag. 2007, 27, 997-1005.

8. Finnveden, G.; Hauschild, M.Z.; Ekvall, T.; Guinée, J.; Heijungs, R.; Hellweg, S.; Koehler, A.; Pennington, D.; Suh, S. Recent Developments in Life Cycle Assessment. J. Environ. Manag. 2009, 91, 1-21.

9. Recently, those approaches are sometimes described simply as "process-LCA" and "IO-LCA", respectively [8]. The IO-based LCA is also described as "environmental input-output (EIO)" LCA [10].

10. Guinée, J.B. Handbook on Life Cycle Assessment. Operational Guide to the ISO Standards; Kluwer Academic Publishers: Dordrecht, The Netherlands, 2002.

11. Suh, S.; Huppes, G. Methods for Life Cycle Inventory of a Product. J. Clean. Prod. 2005, 13, 687-697.

12. Ekvall, T.; Tillman, A.M. Open-Loop Recycling: Criteria for Allocation Procedure. Int. J. Life Cycle Assess. 1997, 2, 155-162.

13. In a precedent literature [12], subdivision and system expansion were referred to as allocation based on "cause-oriented" and "effect-oriented" causalities, respectively. Subdivision is also described as the "partitioning" approach [7]. This approach is more often indicated by "allocation" in the narrow sense.

14. The former approach is also referred to as "substitution" or "subtraction" method. System expansion in the narrow sense sometimes indicates only the latter approach [7].

15. Nakatani, J.; Fujii, M.; Moriguchi, Y.; Hirao, M. Life-Cycle Assessment of Domestic and Transboundary Recycling of Post-Consumer PET Bottles. Int. J. Life Cycle Assess. 2010, 15, 590-597.

16. Ekvall, T.; Finnveden, G. Allocation in ISO 14041-A Critical Review. J. Clean. Prod. 2001, 9, 197-208.

17. Those methods are often abbreviated as "ALCA" and "CLCA" respectively [18].

18. Earles, J.M.; Halog, A. Consequential Life Cycle Assessment: A Review. Int. J. Life Cycle Assess. 2011, 16, 445-453.

19. Ekvall, T.; Weidema, B.P. System Boundaries and Input Data in Consequential Life Cycle Inventory Analysis. Int. J. Life Cycle Assess. 2004, 9, 161-171. 
20. Ekvall, T. A market-based Approach to Allocation at Open-loop Recycling. Resour. Cons. Recycl. 2000, 29, 91-109.

21. Frees, N. Crediting aluminium recycling in LCA by demand or by disposal. Int. J. Life Cycle Assess. 2008, 13, 212-218.

(C) 2014 by the authors; licensee MDPI, Basel, Switzerland. This article is an open access article distributed under the terms and conditions of the Creative Commons Attribution license (http://creativecommons.org/licenses/by/3.0/). 